\title{
Differential Interstrain Susceptibility to Vertebrobasilar Dolichoectasia in a Mouse Model
}

\author{
(D) Y.-Q. Zhu, (D) H. Xing, (DD. Dai, DD.F. Kallmes, and (D) Radirvel
}

\begin{abstract}
BACKGROUND AND PURPOSE: Vetebrobasilar dolichoectasia is characterized by arterial elongation, dilation, and tortuosity and leads to high risks of ischemic stroke. Our aim was to investigate the differential susceptibility to elastase-induced vertebrobasilar dolichoectasia induction in 2 different mouse strains.
\end{abstract}

MATERIALS AND METHODS: Elastase (25 mU) was injected into the cisterna magna in C57BL/6) ( $n=36)$ and 129/SvEv (SV129) ( $n=36)$ mice. Control animals were injected with heat-inactivated elastase ( $n=12$ for each strain). At 3, 7, 14, and 28 days after elastase injection, MICROFIL polymer perfusion was performed. The arterial tortuosity index and the percentage increase in diameter were calculated for the basilar artery. Arterial samples were processed for conventional histologic examination, immunostaining, and matrix metalloproteinase expression. A $\geq 50 \%$ increase in diameter and a tortuosity index of $\geq 10$ for the basilar artery were used to indicate success in achieving vertebrobasilar dolichoectasia.

RESULTS: Successful vertebrobasilar dolichoectasia induction was noted in $67 \%$ (18 of 27 ) of the C57BL/6) strain versus $0 \%$ ( 0 of 19 ) of the SV129 strain $(P<.001)$. Vertebrobasilar dolichoectasia was not observed in sham-operated controls. Both the tortuosity index and diameter increase for the basilar artery were greater in the C57BL/6) strain compared with the SV129 strain $(56.3 \% \pm 16.4 \%$ versus $21.1 \% \pm 21.6 \%$ for diameter, $P<.001 ; 17.4 \pm 7.6$ versus $10.4 \pm 3.8$ for tortuosity index, $P<.001)$. Expression of pro-matrix metalloproteinase- 2 and pro- and active matrix metalloproteinase- 9 was increased in elastase-injected C57BL/6) animals compared with elastase-injected SV129 animals ( $P=$ $.029,0.029$, and 0.029, respectively). Inflammation scores were significantly higher in C57BL/6) animals versus SV129 animals ( $P<.001)$. C57BL/6) subjects demonstrated arterial wall dilation and elongation characterized by internal elastic lamina disruption, muscular layer discontinuity, inflammatory cell infiltration, and high matrix metalloproteinase expression in the media.

CONCLUSIONS: C57BL/6J mice demonstrated greater susceptibility to vertebrobasilar dolichoectasia induction than SV129 mice.

ABBREVIATIONS: $\mathrm{BA}=$ basilar artery; $\mathrm{MMP}=$ matrix metalloproteinase; $\mathrm{TI}=$ tortuosity index; $\mathrm{VBD}=$ vertebrobasilar dolichoectasia

ntracranial arterial dolichoectasia is an arteriopathy characterized by elongation, dilation, and tortuosity of the intracranial vasculature, with the posterior circulation and basilar artery (BA) most frequently affected. ${ }^{1,2}$ Development of vertebrobasilar dolichoectasia (VBD) portends a high risk of subsequent neurologic decline, resulting from ischemic stroke, perforator infarction, and compression of critical posterior fossa structures. Recent, large clinical studies have shown that VBD may be found in up to $12 \%$ of patients presenting with stroke. ${ }^{3}$ Current under-

Received April 29, 2016; accepted after revision October 11.

From the Department of Radiology (Y.-Q.Z., H.X., D.D., D.F.K., R.K.), Mayo Clinic, Rochester, Minnesota; Department of Radiology (Y.-Q.Z.), Shanghai Jiao Tong University Affiliated Sixth People's Hospital, Shanghai, China; and Department of Pathology (H.X.), Shanghai East Hospital, Medical School of Shanghai Tongji University, Shanghai, China.

Please address correspondence to Ramanathan Kadirvel, PhD, Mayo Clinic, 200 First St SW, Rochester, MN 55905; e-mail: kadir@mayo.edu

http://dx.doi.org/10.3174/ajnr.A5028 standing has focused on the role of the internal elastic lamina, the disruption of which, along with medial smooth-muscle atrophy, has been suggested as critical in the progression of the disease. However, the exact pathophysiology of this disorder is poorly understood, ${ }^{4,5}$ and therapeutic options remain limited. Indeed, at present, there is no medical, surgical, or interventional therapy shown to mitigate the progression of VBD. Improved understanding of molecular and genetic features may allow improved treatment paradigms for this often-devastating disorder.

Notwithstanding widely acknowledged limitations of smallanimal models in human disease, aneurysmal disease represents a focused area in which murine models have shown promise. Previous studies of experimental abdominal aortic aneurysms have used numerous lines of research, including assessment of differential susceptibility to aneurysm formation in different mouse strains, to probe the underlying pathophysiology of this disorder. We recently reported a preclinical model, in C57BL/6J mice, of 
VBD induced via microsurgical injection of porcine elastase into the cisterna magna to induce morphologic changes similar to human VBD. ${ }^{6}$ The aims of the current study were to elucidate the morphologic and molecular differences in VBD between C57BL/6J and 129/SvEv (SV129) mouse strains.

\section{MATERIALS AND METHODS \\ Elastase Injection Procedure}

C57BL/6J $(n=48)$ and SV129 $(n=48)$ female mice (6-8 weeks of age) (Charles River Laboratories, Wilmington, Massachusetts) were used in the current study and were divided into a test group ( $n=36$ for each strain) and a control group $(n=12$ for each strain). The use of animals and procedures was reviewed and approved by the Institutional Animal Care and Use Committee in Mayo Clinic.

The mouse model of VBD was induced as previously described. ${ }^{6}$ Using a $10-\mu \mathrm{L}$ micro NanoFil syringe (World Precision Instruments, Sarasota, Florida) with a 36-ga beveled needle, we injected $25 \mathrm{mU}$ of porcine elastase (Worthington Biochemical, Lakewood, New Jersey) in $2.5 \mu \mathrm{L}$ of phosphate-buffered saline $(10 \mathrm{mU} / \mu \mathrm{L})$ into the cisterna magna under a dissection microscope. Control mice were injected with inactivated elastase.

\section{MICROFIL Perfusion and Tissue Harvest}

Animals in the test group of each strain were randomly assigned to 4 subgroups ( $n=8$ at each time point) and were sacrificed at 3, 7, 14 , and 28 days after elastase injection. Control mice $(n=8$ for each strain) were sacrificed at 28 days. The MICROFIL (FlowTech, Cockeysville, Maryland) perfusion was performed at follow-up as described previously. ${ }^{6}$ After MICROFIL perfusion, the whole brain along with the cerebral vascular trees was harvested and fixed in $10 \%$ buffered formalin at room temperature at least 24 hours before imaging. Animals designated for zymography analysis were perfused with saline; the basilar artery was harvested at 14 days and snap frozen in liquid nitrogen $(n=8$ for each strain; $n=4$ for each control and test group).

\section{Morphometric Analysis}

Under the dissecting microscope, visual inspection of the arteries at the skull base was performed to determine the presence of tortuosity and enlargement compared with the control samples. The vascular trees were then photographed by using the MicroPublisher 5.0 RTV camera (QImaging; http://www.qimaging. $\mathrm{com} /$ ) attached to the dissection microscope; Q-Capture Pro 7 software (QImaging) with inner calibration was used to capture the images. Morphometric analysis was performed for the BA. We selected the area of maximal dilation for diameter measurements (exterior side to exterior side) from the MICROFIL-perfused gross images by using Image-Pro Plus software (Media Cybernetics, Bethesda, Maryland). The percentage increase in arterial diameter ([individual measured diameter - average diameter of control samples $] \div$ average diameter of control samples) was determined. The previously validated tortuosity index (TI ${ }^{7}$ was applied to calculate the TI for the current study. TI was defined as the following: [(actual length of the vessel / straight-line length of same vessel -1$) \times 100]$. Successful VBD induction was defined as a $\mathrm{TI} \geq 10$ and $\mathrm{a} \geq 50 \%$ increase in $\mathrm{BA}$ diameter compared with control samples.

\section{Histologic Examination}

Harvested samples were fixed in $10 \%$ neutral-buffered formalin for $>48$ hours, dehydrated by using a graded ethanol series $(70 \%-$ $100 \%$ ), and embedded in paraffin. Transverse sections containing the BA were taken at $4-\mu \mathrm{m}$ thickness and stained with hematoxylin-eosin and elastic fiber stains (Verhoeff-van-Gieson) to assess internal elastic lamina disruption in the media. Serial cross-sections of the BA were used to evaluate inflammation reaction on the basis of inflammation scores. ${ }^{8}$ The score was defined as follows: $0=$ no inflammatory cell infiltration; $1=$ minimal or mild: scant, scattered inflammatory cell infiltration; 2 = patchy but localized or limited inflammatory cells; $3=$ marked, attenuated, diffuse inflammatory cell infiltration. Samples were also immunostained by using the rabbit polyclonal smooth-muscle $\alpha$-actin antibody (1:200; Abcam, Cambridge, Massachusetts), rabbit polyclonal CD45 antibody (leukocyte common antigen; 1:200; Abcam), mouse monoclonal antimacrophage antibody (clone MAC387; 1:200; Thermo Fisher Scientific, Waltham, Massachusetts), rabbit polyclonal matrix metalloproteinase (MMP)-9 antibody (1:100; Abcam), and rabbit monoclonal MMP-12 antibody (clone EP1261Y; 1:200; Abcam) by using the immunofluorescent technique. Double immunofluorescent staining was performed by using anti-CD45 (leukocytes) and antimacrophages to assess whether macrophages were included in leukocytes. Negative controls were performed by omitting primary antibodies. Statistical analysis of the histopathologic images was performed with ImagePro Plus software in at least 5 randomly selected high-power ( $\times 400)$ tubulointerstitial fields from each section. Two pathologists assessed every pathologic index in each section and reached agreement by consensus in a blinded manner.

\section{Gelatin Zymography for MMPs}

Soluble proteins were extracted from the BA of elastase-injected ( $n=4$ for each strain) and heat-inactivated elastase-injected ( $n=$ 4 for each strain) groups at 14-day follow-up. Protein samples (20 $\mu$ g per lane) were separated by $10 \%$ zymogram gel (Bio-Rad Laboratories, Hercules, California). Gels were washed with renaturation buffer (Bio-Rad) for 1 hour and then incubated for 48 hours at $37^{\circ} \mathrm{C}$ in development buffer (Bio-Rad). The gels were stained with $0.5 \%$ Coomassie blue R-250 (Thermo Fisher Scientific). The gelatinolytic bands, representing the activities of MMPs, were scanned and analyzed densitometrically by using ImageJ software (National Institutes of Health, Bethesda, Maryland).

\section{Data Analysis and Statistical Methods}

Animals that had neurologic complications or did not survive for specified follow-up time points were excluded from the analysis, as were the MICROFIL-perfused samples that did not show proper casting as indicated by air bubbles or partial filling in the cast.

Effect of follow-up time and strain on percentage BA increase and TI were assessed with robust analysis of variance by using the Huber M-estimator. Variables were then dichotomized into successful or unsuccessful injections for percentage BA increase and 
Table 1: Success of VBD formation in C57BL/6J and SV129 mouse strains ${ }^{\mathrm{a}}$

\begin{tabular}{|c|c|c|c|c|c|c|c|}
\hline \multirow{2}{*}{$\begin{array}{l}\text { Follow-Up } \\
\text { Time } \\
\text { Point (Days) }\end{array}$} & \multicolumn{3}{|c|}{ C57BL/6J } & \multicolumn{3}{|c|}{ SV129 } & \multirow[b]{2}{*}{$P$ Value } \\
\hline & $\begin{array}{l}\text { Total } \\
\text { No. }\end{array}$ & $\begin{array}{c}\text { No. } \\
\text { Successful }\end{array}$ & $\begin{array}{c}\text { Success Rate } \\
(95 \% \mathrm{Cl})\end{array}$ & $\begin{array}{l}\text { Total } \\
\text { No. }\end{array}$ & $\begin{array}{c}\text { No. } \\
\text { Successful }\end{array}$ & $\begin{array}{c}\text { Success Rate } \\
(95 \% \mathrm{Cl})\end{array}$ & \\
\hline 3 & 7 & 4 & $0.57(0.18-0.90)$ & 5 & 0 & $0.00(0.00-0.52)$ & \\
\hline 7 & 8 & 6 & $0.75(0.35-0.97)$ & 3 & 0 & $0.00(0.00-0.71)$ & \\
\hline 14 & 7 & 4 & $0.57(0.18-0.90)$ & 6 & 0 & $0.00(0.00-0.46)$ & \\
\hline 28 & 5 & 4 & $0.80(0.28-1.0)$ & 5 & 0 & $0.00(0.00-0.52)$ & \\
\hline Total No. & 27 & 18 & $0.67(0.46-0.84)$ & 19 & 0 & $0.00(0.00-0.18)$ & $<.001$ \\
\hline
\end{tabular}

${ }^{a}$ Successful VBD was defined as the percentage increase in basilar artery diameter of $\geq 50 \%$ and a tortuosity index of $\geq 10$. The number and proportion of mice showing successful VBD for each strain and time point are displayed. Ninety-five percent Clopper-Pearson Cls are displayed for each estimate. Proportion of successes was compared between strains using the Fisher exact test.

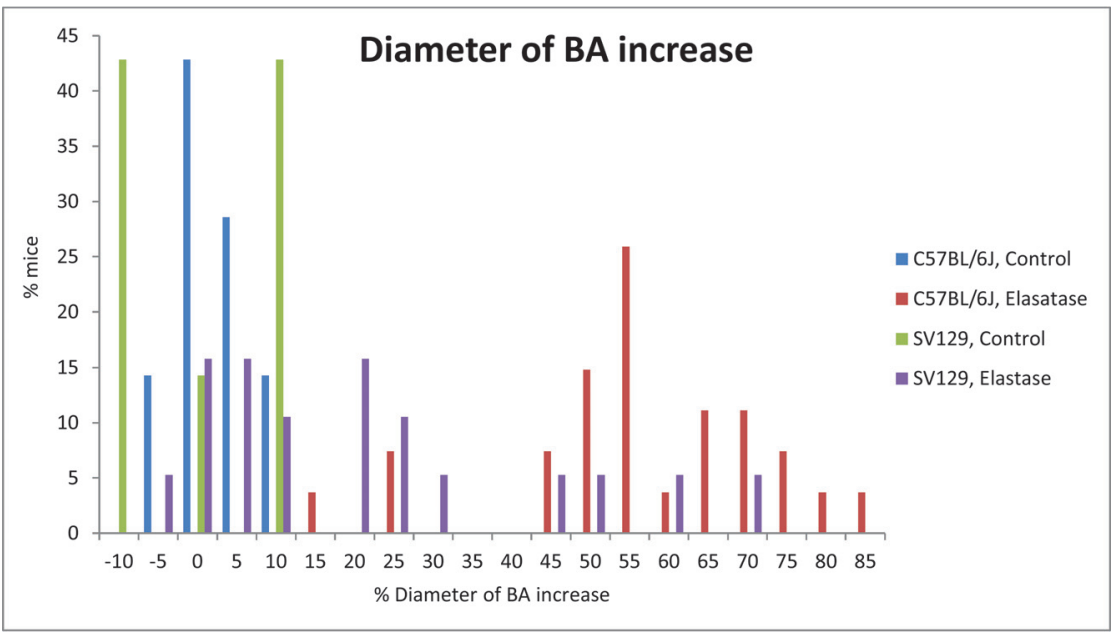

FIG 1. Extent of basilar artery dilation in increased distribution in C57BL/6) and SV129 mouse strains after elastase injection.

TI (with success being defined for BA as a percentage BA increase of $\geq 50$ or for TI as a TI increase of $\geq 10$ ). Results were pooled across time points within strains before between-strain comparisons were performed. The overall proportion of successful VBD (both percentage BA increase of $\geq 50$ and $\mathrm{TI}$ of $\geq 10$ ), proportion of BA increase of $\geq 50 \%$, and the proportion of TI of $\geq 10$ in treated mice were compared by using the Fisher exact test. MMP expression and inflammation scores between strains were compared by using Wilcoxon rank sum tests. Kruskal-Wallis tests were performed to test for the effect of the date of sacrifice on the inflammation score in treated mice, and subsequent pair-wise comparisons were performed by using the Dunn test with a Bonferroni correction. Significance was defined as $P<.05$. Analyses of variance were performed by using SAS (Version 9.3; SAS Institute, Cary, North Carolina). All other analyses were performed by using $\mathrm{R}$ statistical and computing software (Version 3.1.1; http://www.r-project.org/).

\section{RESULTS}

\section{Mortality and Exclusion}

Mortality rates after injection of elastase in C57BL/6J and SV129 mice were $4.2 \%$ (2 of 48 ) and $2.1 \%$ ( 1 of 48 ), respectively ( $P=$ 1.00). Two C57BL/6J mice and 1 SV129 mouse had injectionrelated neurologic complications, including muscle weakness of the left hind leg in 1 and dysequilibrium in 2. No deaths or neurologic complications were observed in the control groups. Three animals in the test group of the C57BL/6 J strain and 12 animals in the test group of the SV129 strain did not show proper MICROFIL polymerization in the BA and were excluded from the analysis.

\section{Macroscopic Examination}

VBD was present in 18 (67\%; 95\% CI, $46.0 \%-83.5 \%)$ of 27 subjects in the C57BL/6J group and none $(0 \%$; 95\% CI, $0 \%-17.6 \%)$ of 19 subjects in the SV129 group $(P<.001)$ (Table 1$)$. VBD was absent in sham-operated control animals of both strains ( $n=8$ for each strain).

Both TI and diameter increases for BA were greater in the C57BL/6J strain compared with the SV129 strain $(56.3 \% \pm$ $16.4 \%$ versus $21.1 \% \pm 21.6 \%$ for diameter; $17.4 \pm 7.6$ versus $10.4 \pm 3.8$ for TI). There was a significant effect of strain on the percentage BA increase and TI $(P<$ $.001)$ and no significant effect of time on the percentage BA increase or TI increase $(P=.69$ and $P=.55$, respectively).

Results were pooled across time points, and the proportion of successful injections was assessed between strains. The proportion of animals with BA increase of $\geq 50 \%$ was significantly higher in C57BL/6J mice at $81 \%$ (22 of $27 ; 95 \%$ CI, $61.9 \%-93.7 \%$ ) compared with $16 \%$ (3 of $19 ; 95 \%$ CI, $3.4 \%-39.6 \%$ ) in the SV 129 strain (Fig $1 ; P<.001$ ). The C57BL/6J strain exhibited TI of $\geq 10$ in $81 \%$ of cases ( 22 of $27 ; 95 \%$ CI, $61.9 \%-93.7 \%$ ) compared with $58 \%$ of cases ( 11 of $19 ; 95 \%$ CI, 33.5\%-79.7\%) in the SV129 strain (Fig 2; $P=.1$ ). The basilar artery diameter increase and TI categorized by time points are presented in Table 2 .

Within strain, the Fisher exact tests showed no significant effect of day of sacrifice on the proportion of successful VBD formation in treated mice of either strain $(P=.98$ and $P=1)$.

\section{Histologic Examination}

Histologic examination showed marked luminal dilation of the BA characterized by internal elastic lamina disruption and a disconnection of the elastic layer in the $\mathrm{C} 57 \mathrm{BL} / 6 \mathrm{~J}$ strain at each time point. CD45-positive cells (monocyte/macrophage) were found in the extravascular space, with some inflammatory cells attached or even infiltrated into the media of the arterial wall. Macrophage staining showed a distribution similar to that of leukocytes. Furthermore, double staining with anti-CD45 and anti-MAC387 revealed that some of the infiltrated inflammatory cells in this model were macrophages (Fig 3). At 14 days, both MMP-9-positive and MMP-12-positive cells were found in the media of the arterial wall in VBD models; MMP-9-positive staining was also found in the infiltrated inflammatory cells (Fig 4).

In the SV129 strain, the internal elastic lamina of the BA appeared to be discontinued, fragmented, and/or it had disappeared 
in the elastase-injected group as that in the C57BL/6J at all follow-up time points. Acute and/or chronic inflammatory cell infiltration was found surrounding and/or within the vessel walls in samples taken at days 3,7 , and 14, but not at day 28 or in control samples. We did not observe positive staining for MMP-9, MMP12, CD45, or MAC387 at any time point.

Inflammation scores in $\mathrm{C} 57 \mathrm{BL} / 6 \mathrm{~J}$ mice were $2.2 \pm 0.84$, $1.67 \pm 0.58,3.0 \pm 0.0$, and $1.5 \pm 0.58$ at $3,7,14$, and 28 days, respectively; in SV129 mice, they were $1.0 \pm 0.82,0.5 \pm 1.0,1 \pm$ 1.41 , and $0.0 \pm 0.0$ at $3,7,14$, and 28 days, respectively. Inflammation scores were significantly different between strains at all time points $(P<.001)$. Within-strain comparisons showed a difference between the dates of sacrifice for the C57BL/6J strain, but not for the SV129 strain $(P=.03$ and $P=.38$, respectively).

\section{Gelatin Zymography}

Gelatin zymography analysis showed that the activities of proand active MMP-2 and MMP-9 were dramatically increased in the VBD samples in C57BL/6J mice compared with sham-operated controls and elastase-injected SV129 mice at 14 days after elastase injection. The activities of pro-MMP-2, active MMP-2, proMMP-9, and active MMP-9 were higher in elastase-injected C57BL/6J groups compared with elastaseinjected SV129 mice and sham-operated C57BL/6J and SV129 groups (Fig 5). Samples from both test and control SV129 mice did not show the activities of either MMP. Wilcoxon rank sum test results were significant for pro-MMP-2 and proand active MMP-9 $(P=.03,0.03$, and 0.03 , respectively), but not active MMP-2 $(P=.07)$.

\section{DISCUSSION}

In the current study, we demonstrated that C57BL/6J mice are markedly more susceptible to VBD induction than SV129 mice. While both mouse strains demonstrated some manifestations of the elongation, dilation, and tortuosity of the intracranial arteries around the circle of Willis, most C57BL/6J mice achieved our prespecified threshold for VBD induction but none of the SV129 mice reached that threshold. In addition, high levels of inflammatory cells and MMPs were observed in the C57BL/6J strain compared with the SV129 strain. Our findings suggest that the inflammatory cascade may be important in the development of VBD and that susceptibility to VBD formation
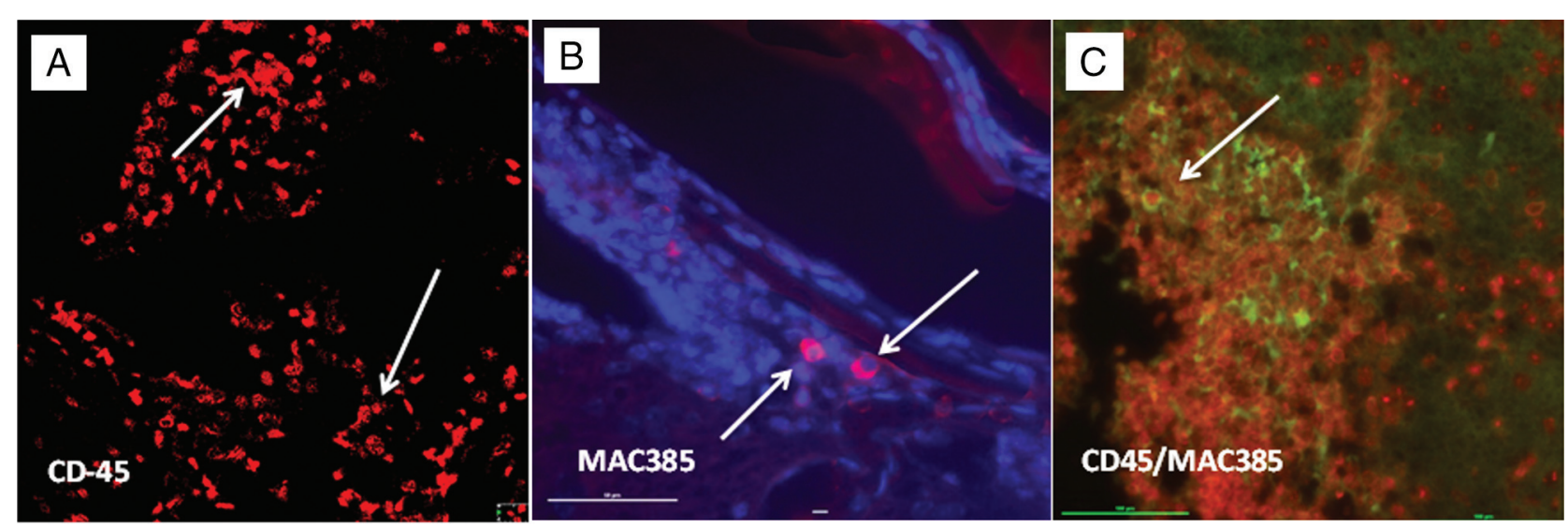

FIG 3. Immunostaining of inflammatory cells in the C57BL/6) mouse strain ( $\times 40$ magnification). Single immunofluorescence staining showing infiltrating CD45+ cells $(A$, arrows) and macrophages ( $B$, arrows). Double immunostaining of infiltrating cells $(C D 45+/ M A C 387+)$ revealed that the infiltrated inflammatory cells were macrophages (C). 

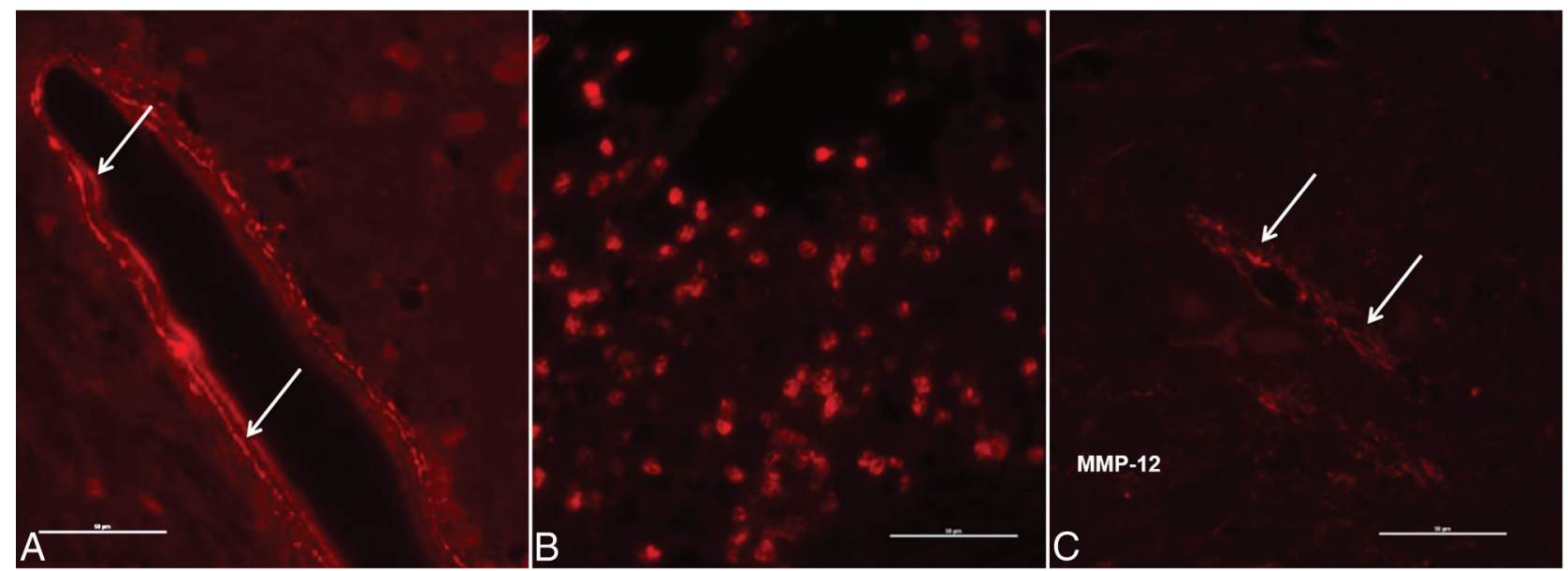

FIG 4. Immunostaining of matrix metalloproteinases in C57BL/6) mouse strain ( $\times 40$ magnification). MMP-9 $(A)$ and MMP-12 (C) were highly expressed in the arterial wall after elastase injection. Elevated expression of MMP-9 was also detected in the infiltrated inflammatory cells $(B)$.

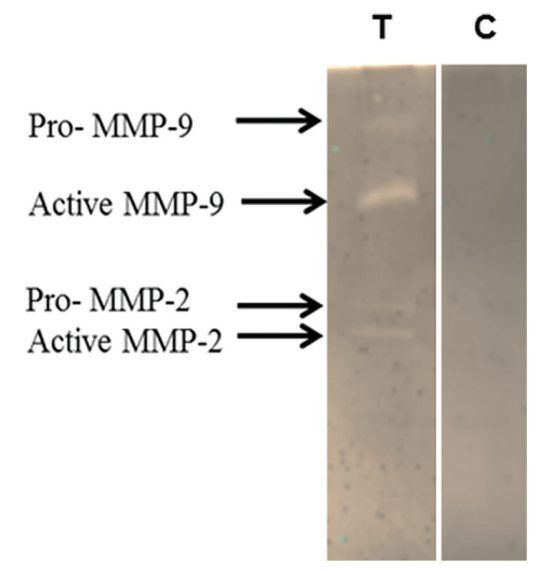

A

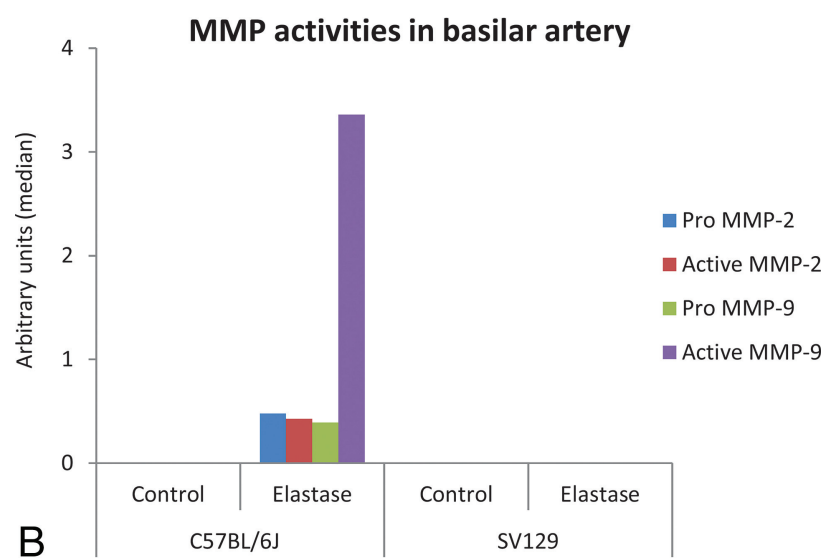

FIG 5. A, Gelatin zymography showing expression of matrix metalloproteinases MMP-2 and MMP-9 in the basilar artery of elastase-injected $(T)$ and heat-inactivated elastase-injected $(C) C 57 B L / 6 J$ mice. $B$, Densitometric analysis of MMP activities in the basilar artery.

after elastase infusion is associated, at least in part, with genetically determined inherited traits. These current findings are important not only in identifying key elements relevant to the development of VBD but also in providing a potential pathway for future discovery of genetic determinants of VBD susceptibility, possibly through linkage analysis of different strains.

The relationship among inflammation, MMPs, and aneurysms has been widely reported in aneurysm models and clinical cases. ${ }^{9-14}$ The disruption of the internal elastic lamina by elastase injection could be the initial factor for induction of VBD. Subsequent infiltration of macrophages and inflammatory cells in the medial layer with secretion of both MMP-9 (collagenase) and MMP-12 (macrophage elastase) followed by degradation of extracellular matrix and elastic lamellae could be leading to the progression of VBD in the C57BL/6J strain. The low level of inflammatory cells and undetectable activities of MMPs in SV129 mice could be associated with less BA dilation and elongation compared with the C57BL/6J strain.

A prospective clinical trial focused on MMP activities, and polymorphism in intracranial arterial dolichoectasia revealed that plasma levels of MMP-2 and MMP-9 were not associated with dolichoectasia. ${ }^{15}$ Furthermore, dolichoectasia was strongly connected to lower levels of MMP-3 and 5A/6A polymorphism of the promoter region of MMP-3. This study was in contradiction to our findings; however, we did not measure MMP levels in plasma. It is possible that specific genetic factors may lead to VBD.

Similar to our own findings, Fujii et $\mathrm{al}^{16}$ revealed that a wide divergence in susceptibility to aneurysmal dilation occurred between C57BL/6J and SV129 mice in an elastase-induced abdominal aortic aneurysm (AAA) model. In particular, C57BL/6J mice were termed "AAA susceptible," and SV129 mice, "AAA resistant." Following elastase perfusion, the SV129 mice had an extent of dilation of $100.0 \%$, whereas the C57BL/6J mice demonstrated an extent of dilation of $156 \%$. The outcross strain between 129 / SvEv and C57BL/6J (ie, B6xSvEv F1 heterozygotes) showed an overall extent of dilation of $150.0 \%$, in between the 2 parent strains. These observations suggest that the susceptibility to form abdominal aortic aneurysms following elastase perfusion is associated, at least in part, with genetically determined inherited traits. It is expected that future investigations based on this information will help define the inherited genetic elements that might influence aneurysmal dilation. Meanwhile, other studies showed differences between these mouse strains in susceptibility to ischemic injury and insulin resistance. ${ }^{16,17}$

Our study has several limitations. In the previous study, the success of achieving the intracranial dolichoectasia was defined as the 
arterial diameter increase of $\geq 25 \%$ compared with controls. ${ }^{6}$ In this article, we applied high stringent and clinically relevant criteria in defining the VBD formation and thus changed the arterial diameter increase from $\geq 25 \%$ to $\geq 50 \%$ on the basis of the criteria of Smoker et al. ${ }^{18}$ The arterial dilation and tortuosity index were calculated by using 2D images; however, we believed that 2D images could well reflect its $3 \mathrm{D}$ tortuosity because we had found that the vertebrobasilar artery mainly goes on the pons and its tortuosity occurred parallel to the surface of pons and in 1 plane. In addition, the pressure perfusion was not performed for MICROFILL polymer injections; instead, manual perfusion was performed. However, extreme care was taken to avoid the variations in the perfusion fixation among animals. In this study, we used a referred dosage for the C57BL/6J strain, which might not be the optimal dosage for the $129 / \mathrm{SvEv}$ strain and could be a partial reason for differences. We performed neither bone marrow transplant experiments nor quantitative loci trait analysis to characterize VBD susceptibility between strains. We did not use any inflammation or MMP inhibitors or gene knockout animals to identify the roles of inflammation and MMPs in the pathogenesis of VBD. We did not observe any MMP expression in the SV129 strain, which could be related to the lower quantity $(20 \mu \mathrm{g})$ of total protein used for zymography experiments. A robust longitudinal study is critical in elucidating the mechanisms of VBD pathobiology.

\section{CONCLUSIONS}

C57BL/6J mice demonstrated greater susceptibility to VBD induction than SV129 mice.

\section{ACKNOWLEDGMENTS}

We express our gratitude to Tina Gunderson, MS, Department of Biomedical Statistics and Informatics, Mayo Clinic, for her generous help with the study.

Disclosures: David F. Kallmes_UNRELATED: Consultancy: Medtronic, Comments: clinical trial planning*; Grants/Grants Pending: MicroVention, Sequent Medical, Surmodics, NeuroSigma, Codman Neuro, Medtronic, Comments: preclinical research and clinical trials*; Travel/Accommodations/Meeting Expenses Unrelated to Activities Listed: Medtronic, Comments: FDA panel meeting presentation. * ${ }^{*}$ Money paid to the institution.

\section{REFERENCES}

1. Pico F, Labreuche J, Touboul PJ, et al. Intracranial arterial dolichoectasia and small-vessel disease in stroke patients. Ann Neurol 2005; 57:472-79 CrossRef Medline

2. Wolters FJ, Rinkel GJ, Vergouwen MD. Clinical course and treat- ment of vertebrobasilar dolichoectasia: a systematic review of the literature. Neurol Res 2013;35:131-37 CrossRef Medline

3. Lou M, Caplan LR. Vertebrobasilar dilatative arteriopathy (dolichoectasia). Ann N Y Acad Sci 2010;1184:121-33 CrossRef Medline

4. Read D, Esiri MM. Fusiform basilar artery aneurysm in a child. Neurology 1979;29:1045-49 CrossRef Medline

5. Sudhir K, Ports TA, Amidon TM, et al. Increased prevalence of coronary ectasia in heterozygous familial hypercholesterolemia. Circulation 1995;91:1375-80 CrossRef Medline

6. Dai D, Kadirvel R, Rezek I, et al. Elastase-induced intracranial dolichoectasia model in mice. Neurosurgery 2015;76:337-43; discussion 343 CrossRef Medline

7. Morris SA, Orbach DB, Geva T, et al. Increased vertebral artery tortuosity index is associated with adverse outcomes in children and young adults with connective tissue disorders. Circulation 2011;124: 388-96 CrossRef Medline

8. Fan XJ, Zhao HD, Yu G, et al. Role of inflammatory responses in the pathogenesis of human cerebral aneurysm. Genet Mol Res 2015;14: 9062-70 CrossRef Medline

9. Tulamo R, Frosen J, Hernesniemi J, et al. Inflammatory changes in the aneurysm wall: a review. J Neurointerv Surg 2010;2:120-30 CrossRef Medline

10. Yoshimura K, Nagasawa A, Kudo J, et al. Inhibitory effect of statins on inflammation-related pathways in human abdominal aortic aneurysm tissue. Int J Mol Sci 2015;16:11213-28 CrossRef Medline

11. Ishibashi R, Aoki T, Nishimura M, et al. Contribution of mast cells to cerebral aneurysm formation. Curr Neurovasc Res 2010;7:113-24 CrossRef Medline

12. Kanematsu Y, Kanematsu M, Kurihara C, et al. Critical roles of macrophages in the formation of intracranial aneurysm. Stroke 2011;42: 173-78 CrossRef Medline

13. Aoki T, Kataoka H, Moriwaki T, et al. Role of TIMP-1 and TIMP-2 in the progression of cerebral aneurysms. Stroke 2007;38:2337-45 CrossRef Medline

14. Nuki Y, Tsou TL, Kurihara C, et al. Elastase-induced intracranial aneurysms in hypertensive mice. Hypertension 2009;54:1337-44 CrossRef Medline

15. Pico F, Jacob MP, Labreuche J, et al. Matrix metalloproteinase-3 and intracranial arterial dolichoectasia. Ann Neurol 2010;67:508-15 CrossRef Medline

16. Fujii M, Hara H, Meng W, et al. Strain-related differences in susceptibility to transient forebrain ischemia in SV-129 and C57black/6 mice. Stroke 1997;28:1805-10; discussion 1811 CrossRef Medline

17. Almind K, Kahn CR. Genetic determinants of energy expenditure and insulin resistance in diet-induced obesity in mice. Diabetes 2004;53:3274-85 CrossRef Medline

18. Smoker WR, Corbett JJ, Gentry LR, et al. High-resolution computed tomography of the basilar artery, 2: vertebrobasilar dolichoectasia: clinical-pathologic correlation and review. AJNR Am J Neuroradiol 1986;7:61-72 Medline 